NASA/CR-2000-209955

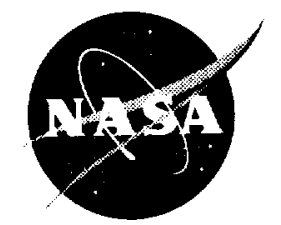

\title{
A New Clinical Instrument for the Early Detection of Cataract Using Dynamic Light Scattering and Corneal Topography
}

Rafat R. Ansari

National Center for Microgravity Research, Cleveland, Ohio

Manuel B. Datiles, III

National Eye Institute/NIH, Bethesda, Maryland

James F. King

Dynacs Engineering Company, Inc., Brook Park, Ohio

Prepared under Cooperative Agreement NCC3-544

National Aeronautics and

Space Administration

Glenn Research Center 


\section{Acknowledgments}

This work was performed under a NASA-NIH Interagency Agreement.

Available from

NASA Center for Aerospace Information 7121 Standard Drive

Hanover, MD 21076

Price Code: A03
National Technical Information Service 5285 Port Royal Road Springfield, VA 22100 Price Code: A03 


\title{
A NEW CLINICAL INSTRUMENT FOR THE EARLY DETECTION OF CATARACT USING DYNAMIC LIGHT SCATTERING AND CORNEAL TOPOGRAPHY
}

\author{
Rafat R. Ansari \\ National Center for Microgravity Research in Fluids and Combustion \\ Cleveland, Ohio 44135 \\ Manuel B. Datiles III \\ National Eye Institute/NIH \\ Bethesda, Maryland 20892 \\ James F. King \\ Dynacs Engineering Company, Inc. \\ Brook Park, Ohio 44142
}

\begin{abstract}
A growing cataract can be detected at the molecular level using the technique of dynamic light scattering (DLS). However, the success of this method in clinical use depends upon the precise control of the scattering volume inside a patient's eye and especially during patient's repeat visits. This is important because the scattering volume (cross-over region between the scattered light and incident light) inside the eye in a high-quality DLS set-up is very small (few microns in dimension). This precise control holds the key for success in the longitudinal studies of cataract and during anti-cataract drug screening. We have circumvented these problems by fabricating a new DLS fiber optic probe with a working distance of $40 \mathrm{~mm}$ and by mounting it inside a cone of a corneal analyzer. This analyzer is frequently used in mapping the corneal topography during PRK (photorefractive keratectomy) and LASIK (laser in situ keratomileusis) procedures in shaping of the cornea to correct myopia. This new instrument and some preliminary clinical tests on one of us (RRA) showing the data reproducibility are described.
\end{abstract}

\section{INTRODUCTION}

Cataract is a leading cause of blindness all over the world. Since this disease has no medical cure except the surgical removal of the opacified lens, it is proving to be a major public health burden. It is estimated that more than 70 million Americans will have cataract in year 2030 compared to 34 million figure today. The problem in Africa and India is already out of control with cataract extractions taking place at relatively young age (in the $40^{\prime} \mathrm{s}$ ). Foster ${ }^{\prime}$ estimates that in 20 years, in spite of surgical efforts by ophthalmologists, and because of the aging of the world population, there will be 200 million eyes legally blind worldwide from cataract. The social repercussions of visual impairment due to cataract are growing. In a recent Salisbury Eye Evaluation (SEE) project, Rubin ${ }^{2}$ has shown that $1 / 3$ of all vision impairment in patients ( $\geq 65$ years) is due to cataract. This could lead to dependency on others, reduced physical activity, social isolation, and even death. For example in many communities in the United States driving an automobile is the primary means of transportation and is considered an integral part of one's mobility and therefore quality of life. Recently Owsley ${ }^{3}$ linked cataract as the leading cause of vision impairment in older adults, affecting almost half of those over 75 years of age. She estimates that older drivers are the fastest growing group of drivers in the United States. By the year 2024 one out of every four drivers on the road will be over the age of 65 , and for every 100,000 miles driven, the crash rate of older drivers is twice than that of younger drivers. Thus the need to control this disease is much greater today than ever before. Kupfer ${ }^{4}$ estimates that a delay in cataract formation of about 
10 years would reduce the prevalence of visually disabling cataract by about $45 \%$. This scenario has raised enthusiasm and optimism among various groups of biochemists, e.g., suggesting both high-tech and low-tech solutions such as the use of drugs like glutathione, pentethine, aldose reductase inhibitors, vitamin supplements, and drinking red wine and (green) tea. But the methods in clinical use for quantifying cataract remain quite primitive and at best elusive at the level of early detection. These include Snellen acuity charts, contrast sensitivity tests, visual function questionnaires, examinations under direct and indirect ophthalmoscopes, slit-lamps, Scheimpflug, and retroillumination imaging. In 1988, based on a study of 1350 patients observed under a slit-lamp apparatus, LOCS (lens opacification classification system) was developed to standardize the photographs of the lens. Later, Chylack et al ${ }^{5}$. developed LOCS-II which gives four standards for analyzing nuclear region. cortical and subcapsular regions, and one standard for the brunescence (color) in the nuclear region of the lens. In 1996, World Health Organization (WHO) simplified the cataract grading system by proposing three standards for nuclear opalescence. But to this date the methods described above have failed to detect and grade cataracts reliably and quantitatively at their early stages of formation and hence no accurate method is available to test the efficacy of possible medical cure for cataracts. Benedek et al. ${ }^{6}$, were the first to use QELS (quasi-elastic light scattering) to study cataractogenesis, using conventional optical setups. Ansari et al. ${ }^{7}$, and Rovati et al. ${ }^{\gamma}$, have described newly developed fiberoptic based compact and extremely sensitive instrumentation for the early detection of cataracts, vitreous modalities, and corneal structures using DLS methodology. In these instruments DLS was used in slit-lamps and Scheimpflug cameras. Recently Ansari et al $^{9}$. have shown that a growing cataract can be detected using DLS at the molecular level; much earlier than any state-of-the-art clinical methods in use today. However, the success of these methods in clinical use depends upon the precise control of the scattering volume inside a patients' eye and especially during patients' repeat visits. This is of great importance in the longitudinal studies of cataract and during anti-cataract drug screening. This is important because the scattering volume inside the eye is very small (few microns) in dimension. Slight variation in the measurement site inside a patient's lens can cause significantly different values in measured parameters of interest. In this paper we have circumvented these problems by fabricating a new DLS fiber optic probe with a working distance of $40 \mathrm{~mm}$ and by mounting it inside a cone of a comeal analyzer.

\section{EXPERIMENTAL SET-UP}

A corneal analyzer (Optikon 2000 Keratron, Italy) was modified by placing a DLS fiber optic imaging probe inside its cone as shown in Figure 1. Figure 2 is a schematic illustration of the optical system. The probe design and its general description has been given earlier by Ansari et al ${ }^{10}$. The major difference here is that the probe used in this study has a focal length of $40 \mathrm{~mm}$ from its front surface. This allows plenty of room to work with and an enormous comfort to a patient during the measurements without touching the sensitive corneal tissue. A further advantage is the alignment feature of this device. The front-end eye piece is equipped with two fiber optic position sensors on extreme (equatorial) ends. An infrared (IR) beam of light passes through one fiber and collected by another. When this IR beam is intercepted by the cornea (see figures 1 and 2) the instrument is aligned with the apex of the cornea.

The IR beam does not pass through any other ocular tissue. Thus the actual laser light used during the 2-5 second DLS measurement is never used to align the instrument, making it much safer for human use. The laser light ( $\lambda=665 \mathrm{~nm}$, Power $80 \mu \mathrm{w})$ is transmitted by a compact backscatter fiber optic probe to the eye. The Brownian motion of the protein crystallines inside the lens is monitored. The scattered signal is guided back to a photodetector through the probe where it is amplified. discriminated, and converted into TTL logic pulses. A fiber optic probe comprising two monomode optical fibers and two micro lenses. as illustrated in Figure 2, provides a compact and remote means of studying the dynamical characteristics of the macromolecules in the eye. The probe has a length to width dimension of $3.175 \mathrm{~cm} \mathrm{x} 3.175 \mathrm{~cm}$. The fiber optic probe contains the necessary optics to perform DLS measurements at a scattering angle of $154^{\circ}$. Two monomode optical fibers, each housed in a stainless steel ferrule, are mounted into a separate stainless steel housing. An air gap is intentionally left between the fiber housing and the lens housing in order to produce a tightly focused spot in the scattering volume. The two optical fibers in their housings are aligned and fixed into position off-axis with the micro lens. The two housings are placed 


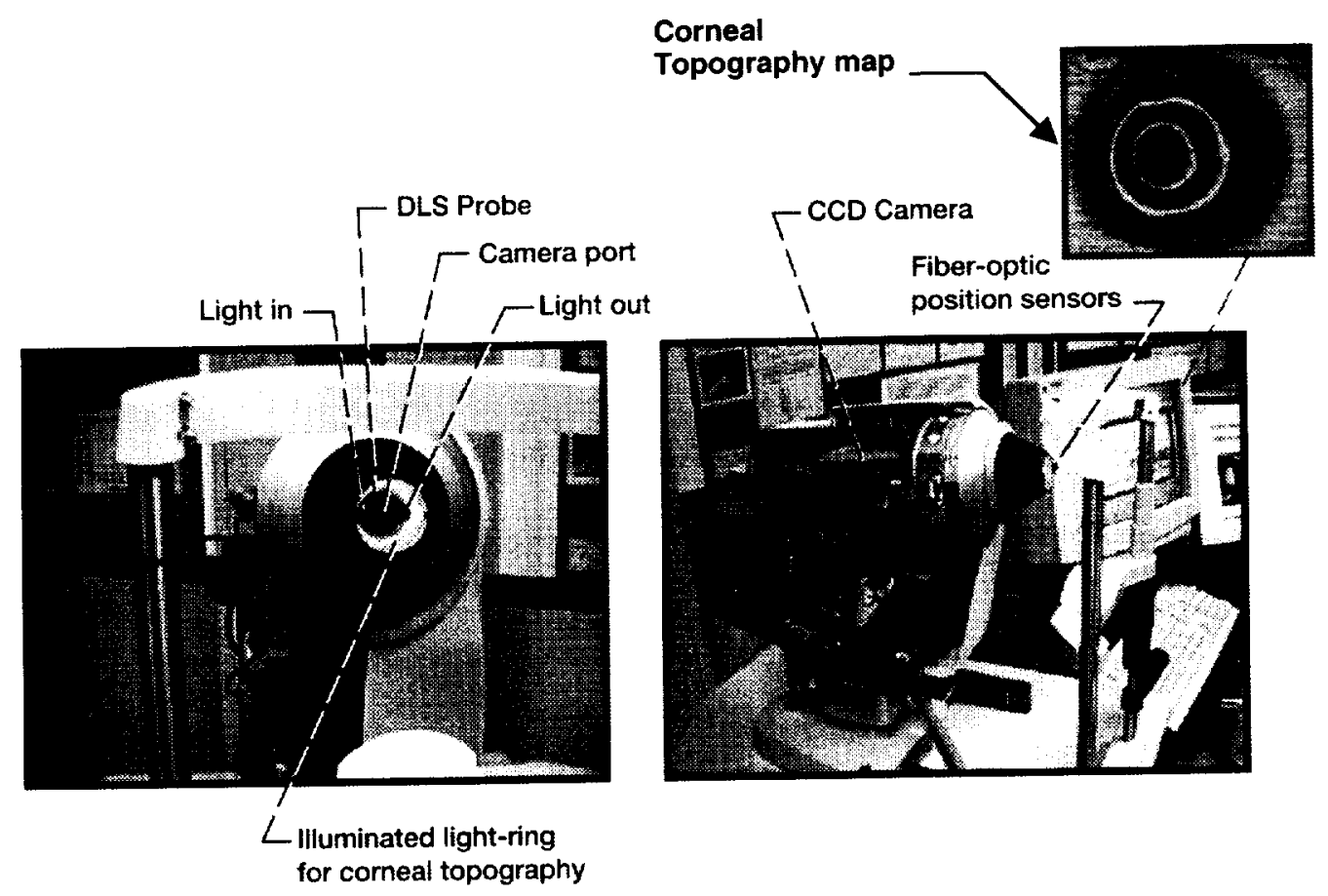

Figure 1. The front and cut-away view of a modified comeal analyzer (Keratron) with DLS probe.

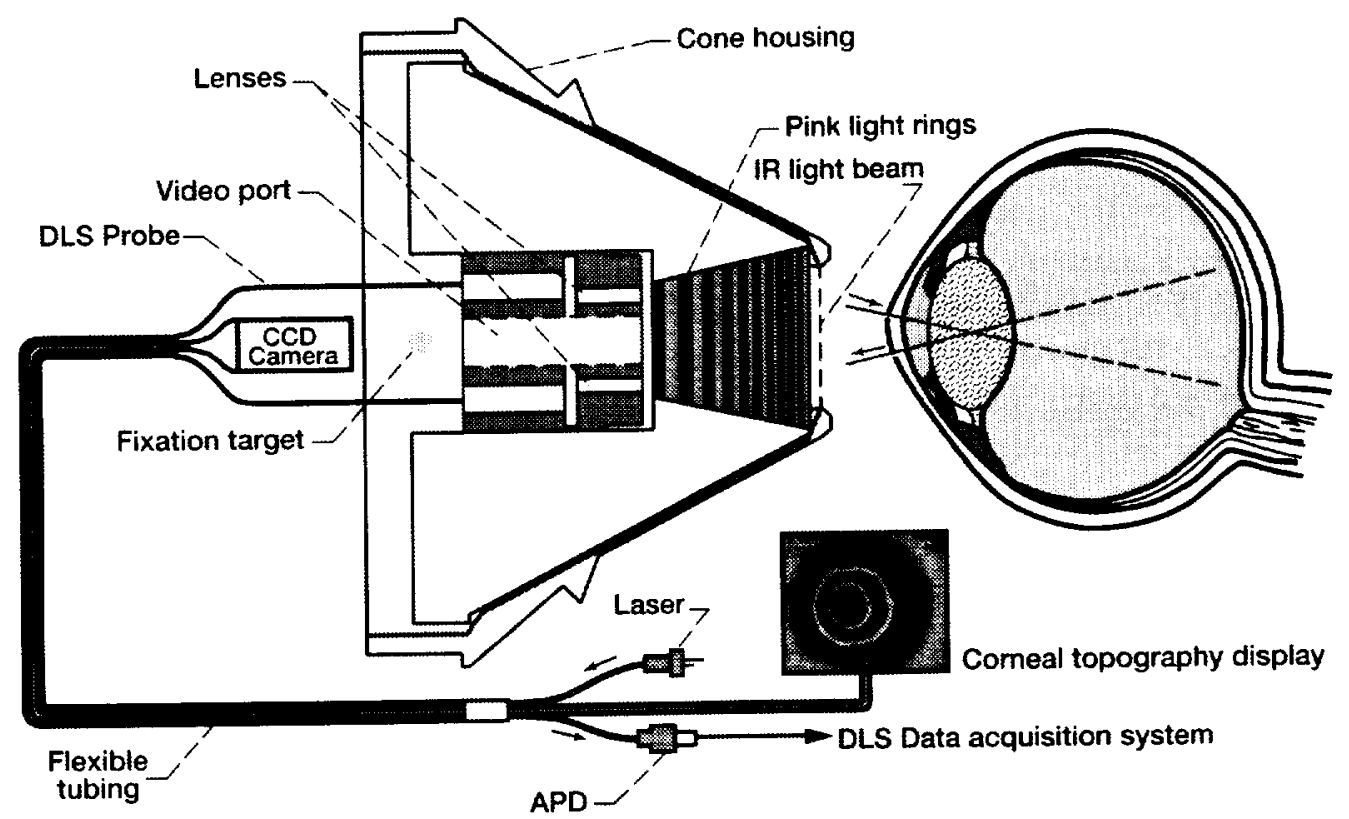

Figure 2. Schematic diagram of the optical system. 
inside a third (outer) housing made of stainless steel, and the back end of the housing is covered with a heat- shrink tubing. The two free ends of the optical fibers were terminated with FC/PC-type male connectors for easy mating with the laser/detector module, containing the laser and the APD (avalanche photo diode) photodetector.

The Keratron Corneal Analyzer utilizes an infra-red detector that pin-points the exact location of the corneal vertex. Detailed description of the comeal geometry reconstruction with this instrument is given by Mattioli and Tripoli". Using the CCD camera located in the center of the cone (Figure 2), an operator, using a joy stick, centers the cone with the optical axis of the eye to view the active image of the patients' eye on the instrument's built-in CRT screen as shown in Figure 3 . While depressing the foot switch, the IR detection system automatically sends a signal to the computer instantaneously grabbing the image when the desired position of the cone has been achieved. The operator simultaneously depresses the "Remote DLS" button, which in turn activates the laser and starts the digital autocorrelator for the DLS measurement. The patient reports seeing a defused speckle light pattern during the 5 second DLS measurement.

The laser and the data acquisition program has been pre set to run for either a 2 second or 5 second measurement duration. When these two operations have been completed, the operator can save and view both the DLS data as well as the corneal mapping data. The operation sequence is shown in Figure 4.

\section{DATA ANAL YSIS}

In our experiments, visible light of $665 \mathrm{~nm}$ wavelength from a laser diode (power $80 \mu \mathrm{w}$ ) is focused into a small scattering volume inside the patient's lens. The detected signal is processed via a digital correlator to yield a time autocorrelation function (TCF). For dilute dispersions of spherical particles the slope of the autocorrelation function provides a quick and accurate determination of the particle's translation diffusion coefficient, which can be related to its size via a Stokes-Einstein equation, provided the viscosity of the suspending fluid, its temperature, and its refractive index are known. The self-beating experiments reported here measure a normalized intensity-intensity temporal autocorrelation function $g^{2}(\tau)$ which is related to the normalized electric field temporal autocorrelation function of the fluctuations in the scattered light amplitude $g^{\prime}(\tau)$ via the Siegert relation,

$$
g^{2}(\tau)=A\left[1+\beta\left|g^{\prime}(\tau)\right|^{2}\right]
$$

where $A=<i>2$ is the average D.C. photocurrent or the baseline of the autocorrelation function, and $\beta(0<\beta<1)$ is an empirical experimental constant and is a measure of the spatial coherence of the scattering geometry of the collection optics which can be related to signal-to-noise $(\mathrm{S} / \mathrm{N})$ and hence considered a measure of the efficiency of a DLS spectrometer. Earlier studies of protein diffusivity in eye lenses by Benedek et al ${ }^{12}$. have established the presence of two decay constants. Equation 1 can be re-written in terms of a fast and a slowly decaying component due to alpha crystalline diffusion and its larger aggregates respectively,

$$
g^{\prime}(\tau)=I_{\alpha} \exp \left(-D_{\alpha} q^{2} \tau\right)+I_{\alpha A} \exp \left(-D_{\alpha A} q^{2} \tau\right)
$$

where $\mathrm{I}_{\alpha}$ and $\mathrm{l}_{\alpha A}, \mathrm{D}_{\alpha}$ and $\mathrm{D}_{\alpha A}$ are the scattering strengths, and corresponding translational diffusion coefficients due to small $\alpha$ crystallines and their larger aggregates in an eye lens respectively and $q=[(4 \pi n / \lambda) \operatorname{Sin}(\theta / 2)]$ is the magnitude of the scattering vector. The Stokes-Einstein relation $[D=K T / 6 \pi \eta R]$ together with the knowledge of the scattering strengths from each size species leads to a particle size distribution. For the human lens the viscosity ( $\eta=0.6915$ centipoise) and a refractive index $(n)$ of 1.333 at a body temperature of approximately $37^{\circ} \mathrm{C}$ was used to extract macromolecular sizes. The autocorrelation functions of the human lens were analyzed using the commercial data inversion routines supplied by the Brookhaven Instruments Corporation (Holtsville, NY). These include a double exponential and an exponential sampling program. 


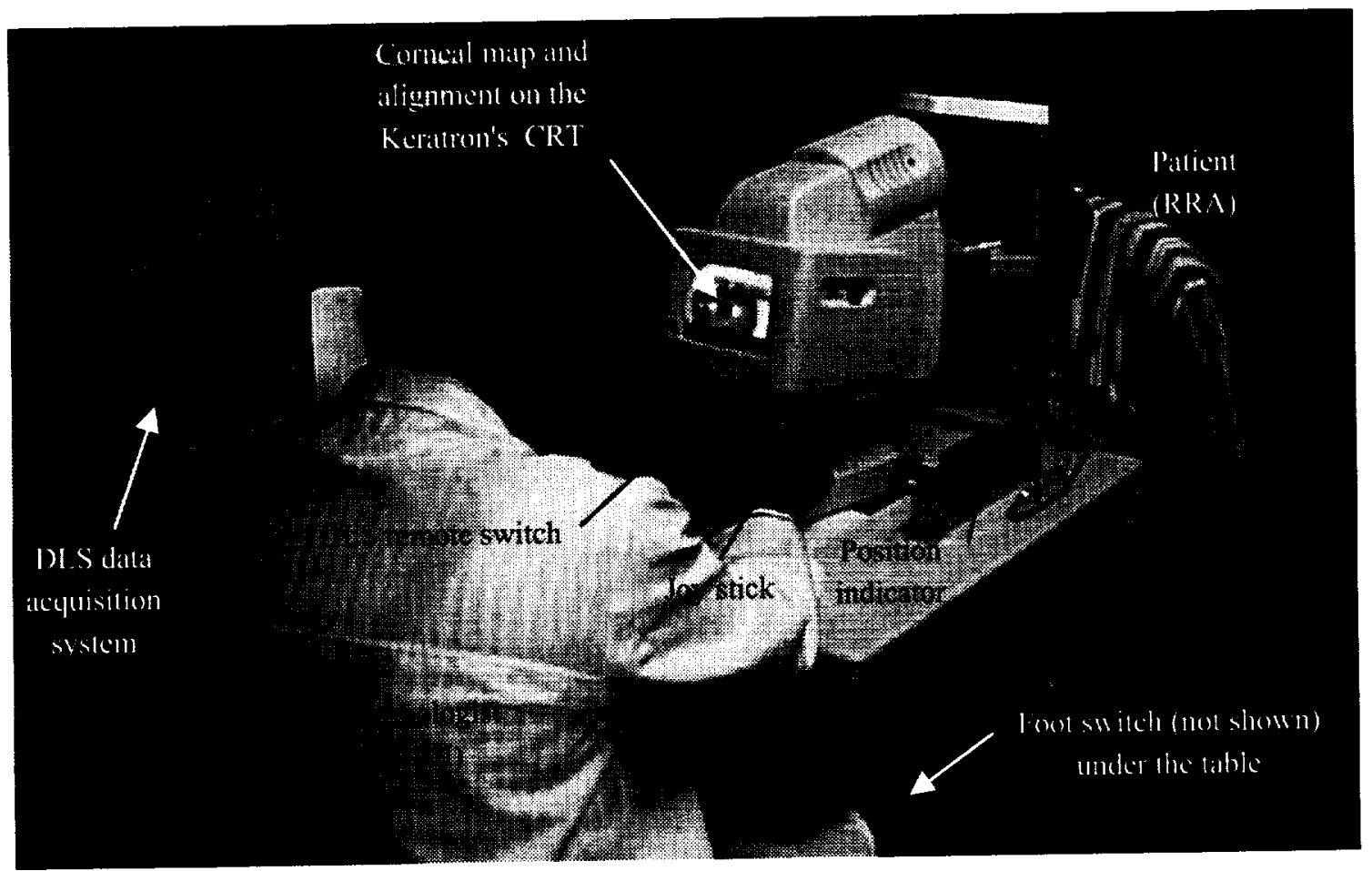

Figure 3. Instrument in clinical operation

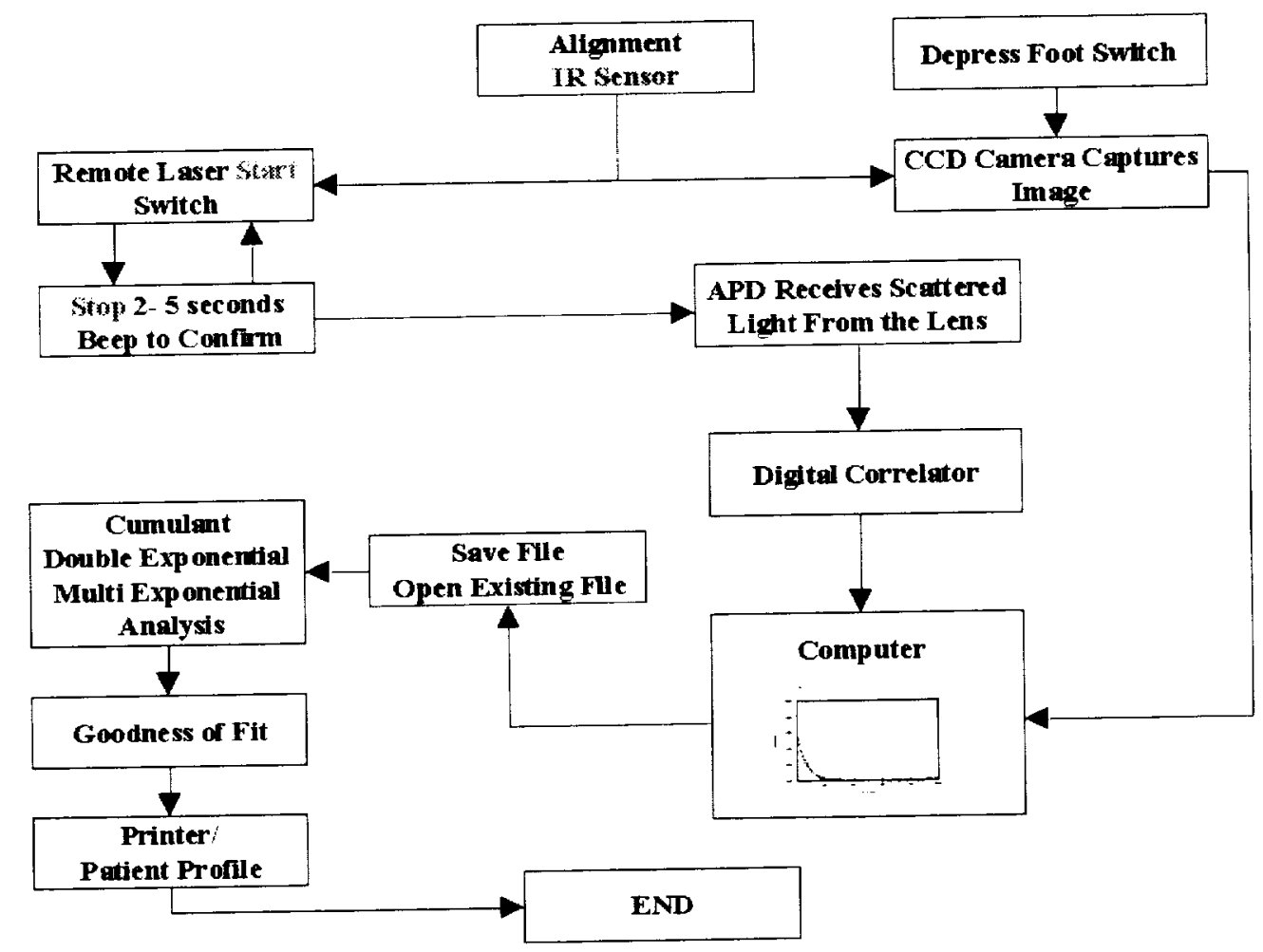

Figure 4. Data acquisition and analysis block diagram 


\section{RESULTS}

\subsection{Scheimpflug Imaging}

We studied both eyes of a 48 year old volunteer (RRA) under a National Eye Institute Intramural Review Board approved clinical research protocol for preliminary testing of the new device. Informed consent was obtained and a complete eye examination was performed including a dilated slit lamp eye examination. This confirmed that both eyes were normal with clear corneas and lenses (LOCS-II nuclear color and opalescence of grade 0 ). Scheimpflug imaging was performed using the Zeiss Scheimpflug camera and NEI Scheimpflug image analysis system described by Datiles et al ${ }^{13}$. This is shown in Figure 5. The mean nuclear densities for both left and right eyes were found to be 0.02 ODU (optical density units). These are within normal limits. The lens thickness (anterior-posterior) for the right and left eyes were found to be $3.96 \mathrm{~mm}$ and 3.92 $\mathrm{mm}$ respectively, both also within normal limit.

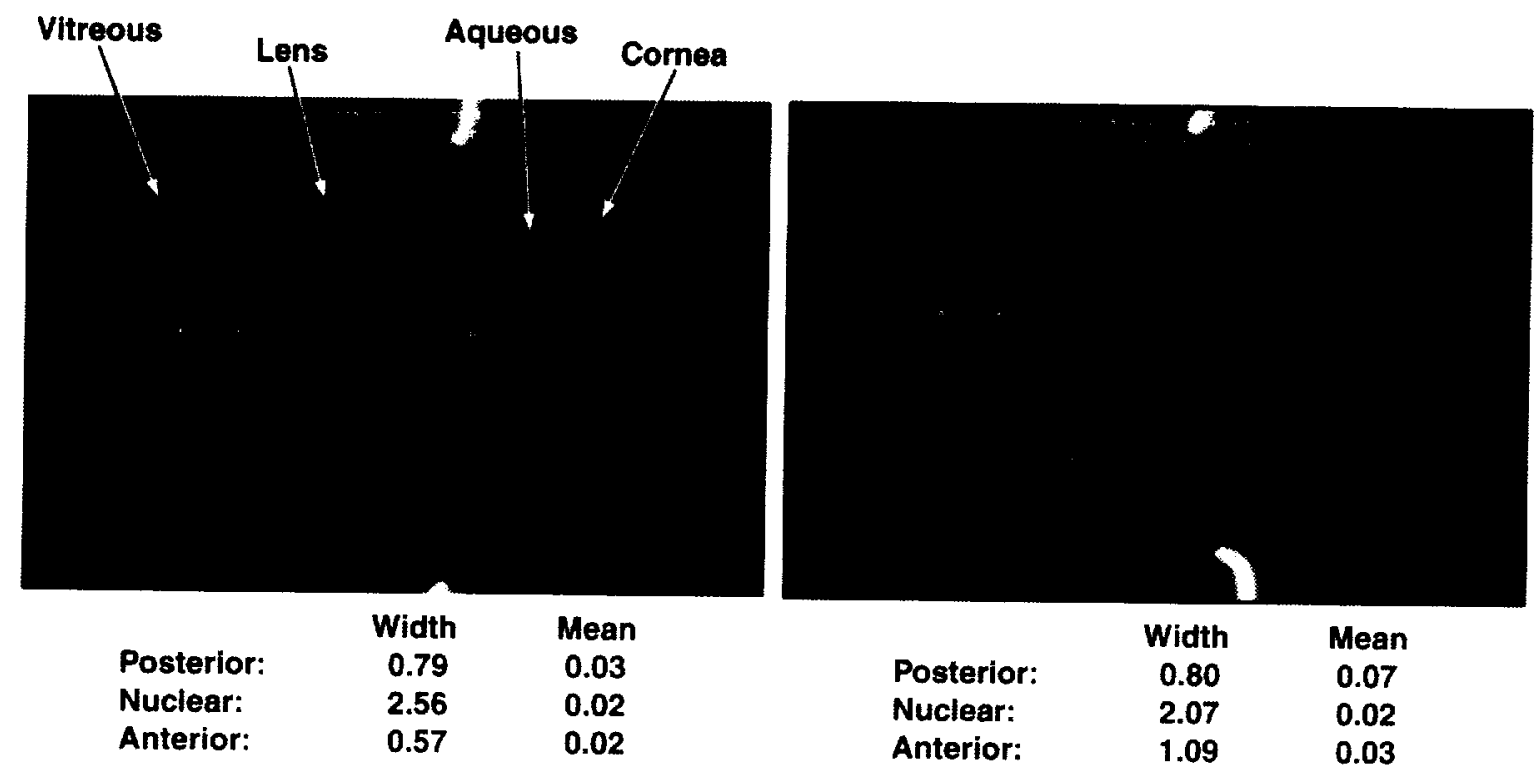

Figure 5. Scheimpflug image of left and right eye

\subsection{Measurement of Ocular dimension with ultrasound}

The corneal tissue is the highest refractive ocular tissue and can be detected very easily by its refractive properties. Unfortunately the transparent nature of the lens and vitreous makes it very difficult to place any markers for position referencing. We therefore measured the ocular chamber dimension with an ultrasound probe (OcuScan by Alcon Biophysics) to make sure of the position in the nuclear region of the lens tissue scanned by the DLS probe. Figure 6 shows this measurement. The axial length (along the optical axis) of the right eye was found to be $22.22 \pm 0.25 \mathrm{~mm}$. It is further broken down in terms of anterior chamber (cornea and aqueous $=2.41 \mathrm{~mm}$ ), lens $(4.18 \mathrm{~mm})$, and vitreous $(15.63 \mathrm{~mm})$. The left eye was found to have an axial length of $23.02 \pm 0.14 \mathrm{~mm}$, anterior chamber being $(2.99 \mathrm{~mm})$, lens $(4.06 \mathrm{~mm})$, and the vitreous $(15.97 \mathrm{~mm})$. 


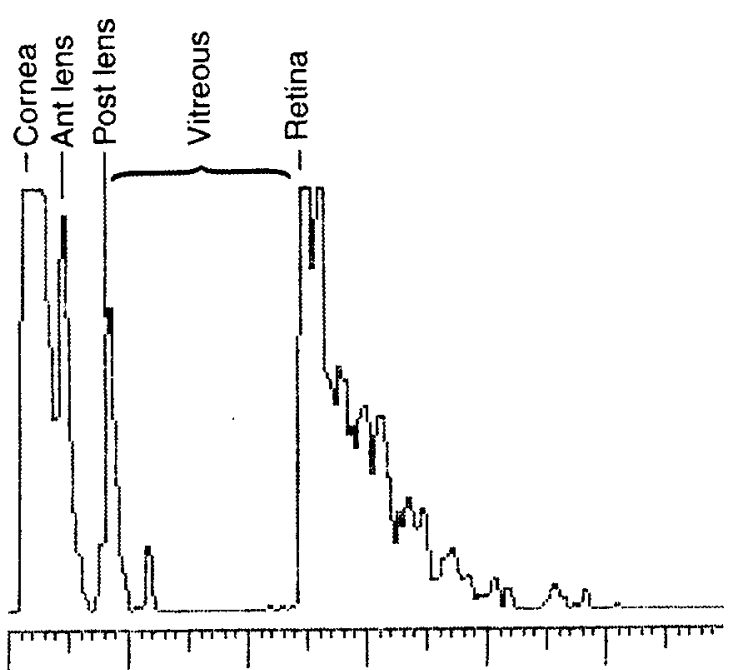

PHAKIC

AVERAGE VALUE: $23.07 \mathrm{~mm}$ AXIAL LENGTH: $\quad 23.02 \mathrm{~mm}$ $A C=2.99 \mathrm{~mm} \quad L=4.06 \mathrm{~mm} \quad V=15.97 \mathrm{~mm}$ a) Left Eye

SD: 0.14

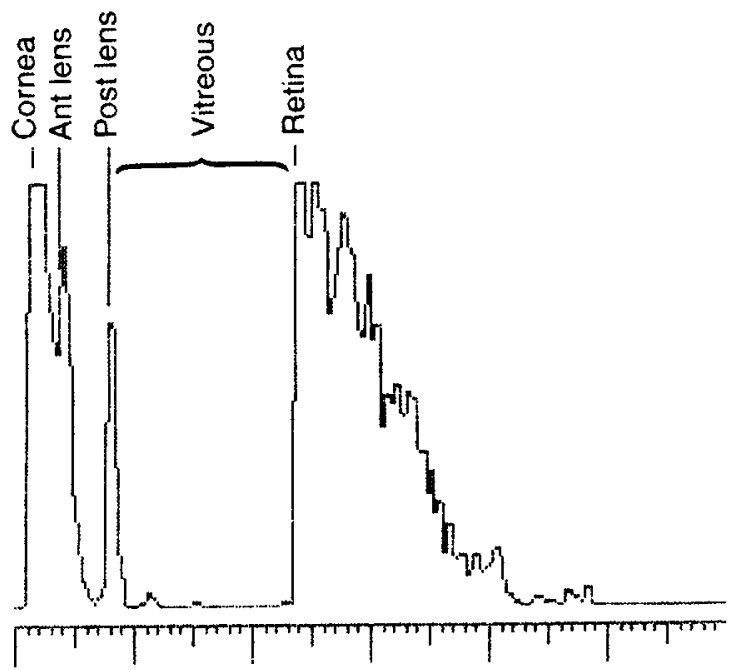

PHAKIC

AVERAGE VALUE: $22.37 \mathrm{~mm}$ AXIAL LENGTH: $\quad 22.22 \mathrm{~mm}$ $A C=2.41 \mathrm{~mm}$ $\mathrm{L}=4.18 \mathrm{~mm}$

SD: 0.25

b) Right Eye

Figure 6. A-scan (ultrasound) measurements.

\subsection{Corneal Morphology}

We also studied the morphology of the corneal endothelium by measuring its cell density using the technique of specular microscopy (Model SP8000FA Specular Microscope by Konan Medical Corp.). This endothelial cell count is generally considered an index of corneal health. The measurements are shown in Figure 7. The corneas were found to be in excellent health with endothelial cell densities of $2890 \pm 71 \mathrm{cells} / \mathrm{mm}^{2}$ and $2817 \pm 80 \mathrm{cells} / \mathrm{mm}^{2}$ for the right and left eyes respectively. A cell density of greater than 2250 is usually assigned to a healthy (normal) cornea. The corneal thickness was found to be normal: $0.515 \mathrm{~mm}$ and $0.534 \mathrm{~mm}$ for the right and left eyes respectively.
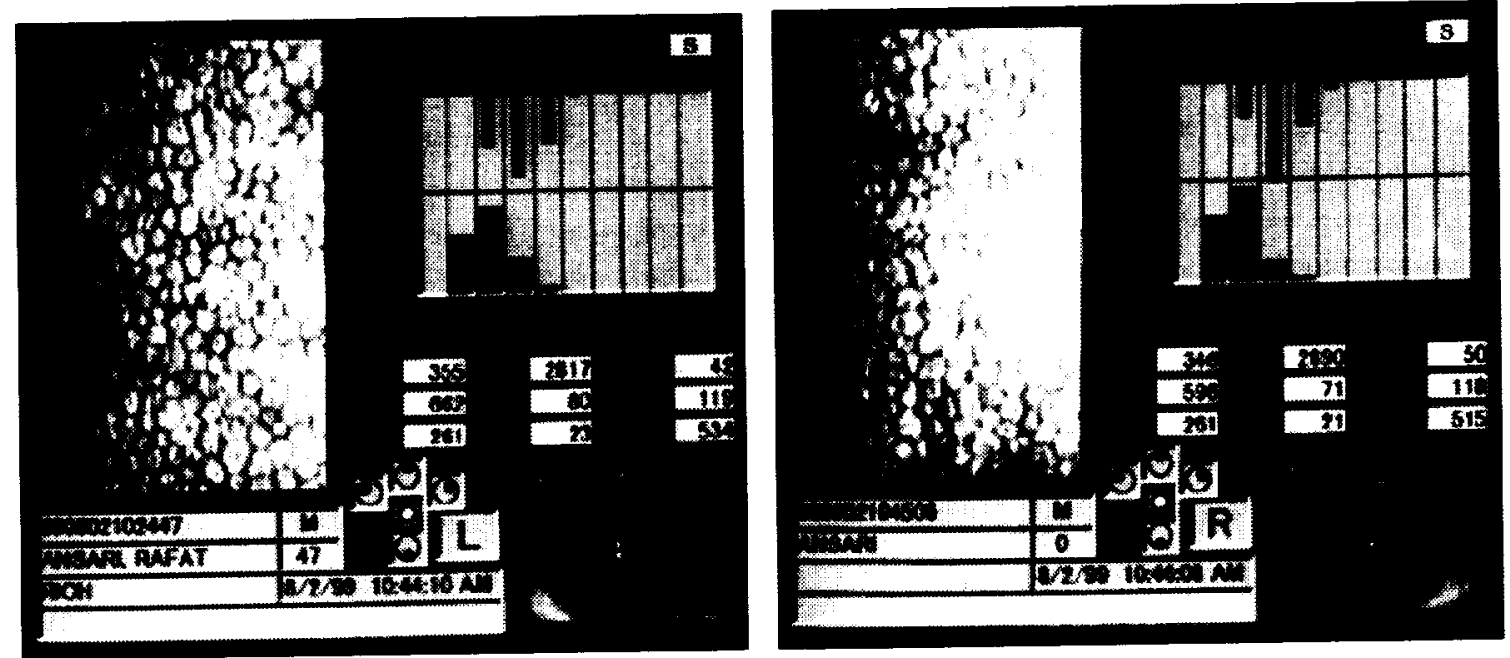

Figure 7. Corneal morphology using specular microscopy. 


\subsection{DLS Measurements}

The eyes were dilated using $1 \%$ mydriacyl and $2.5 \%$ epinephrine eye drops. Only one eye at a time was dilated and used for measurements. The DLS measurements were performed at several different times (or sittings). In order to test the reproducibility of DLS measurements during patient's return visits, after each measurement, the patient was released and brought back after being away from the examination room from several minutes to several hours. A few of these typical measurements (time auto correlation functions or TCF`s) for the left eye are presented in Figure 8.

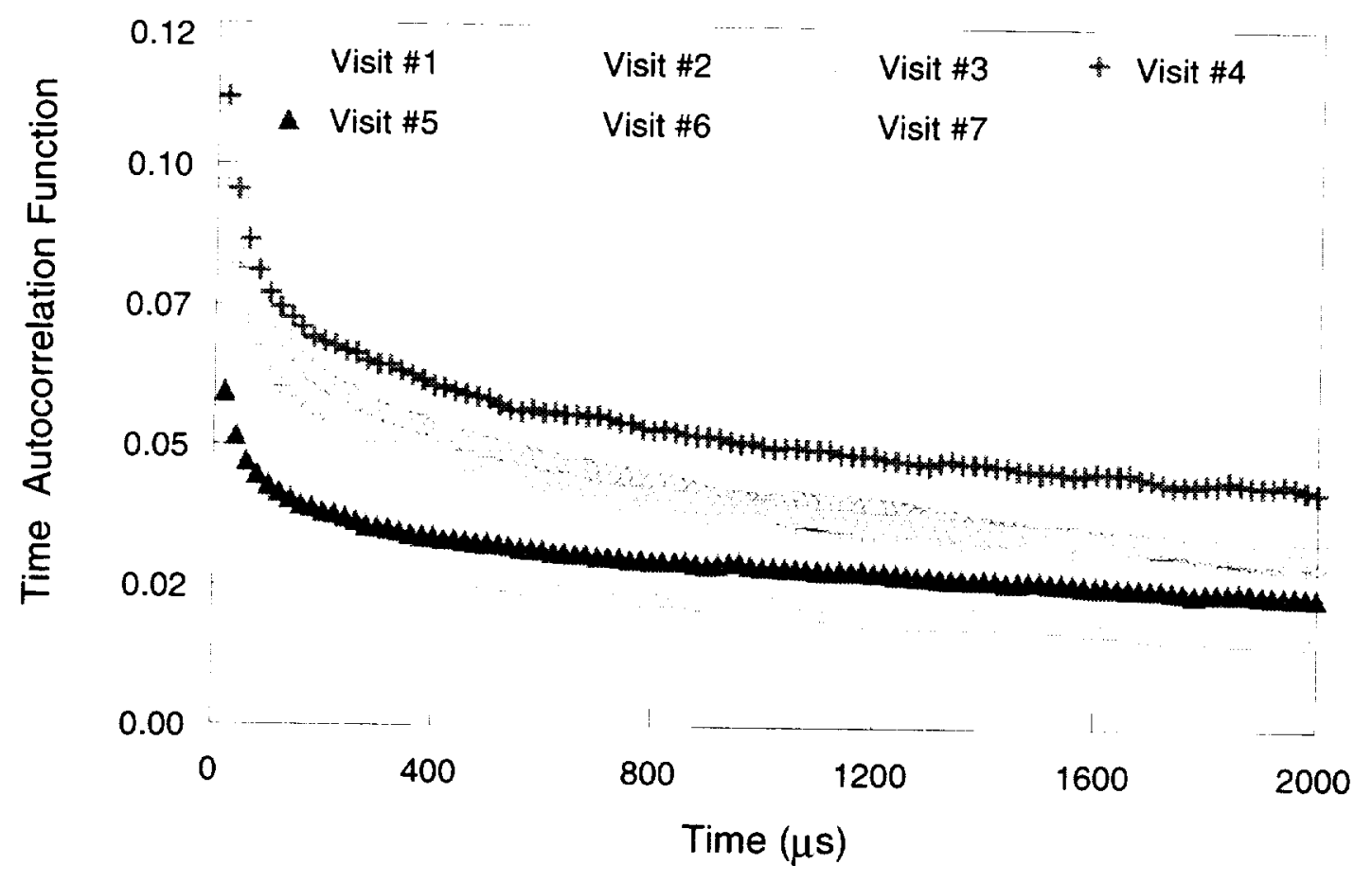

Figure 8. Clinical Instrument Data Repeatability Tests (Patient Return Visits)

Time autocorrelation function (TCF) Profiles.

A quick glance of this data indicates that the slopes of these curves (a measure of diffusion coefficient or particle size) remain constant. However, the baseline values do vary slightly. But, as shown below, this difference is small and does not affect the final outcome. The data is analyzed using the technique of double exponential (eqn. 2) and results are presented in Figure 9. The fast decaying component represents the diffusing motion of alpha crystallines and the slower component represents larger aggregates and/or protein complexes. The fast component diameter is found to be very consistent in different sittings with a value of $41.14 \pm 2.85 \mathrm{~nm}$ (within $7 \%$ ). The slower component is found to be $3.08 \pm 0.52 \mu \mathrm{m}$ (within $17 \%$ ). These uncertainties in the reproducibility of DLS lens data is very good considering the various variables at play in such a measurement, e.g., the statistical nature of the scattered photons, the polydisperse nature of the lens, scattering from lens fibers, patients' fixation on a target, breathing, etc. This point is further described below in a model experiment. 

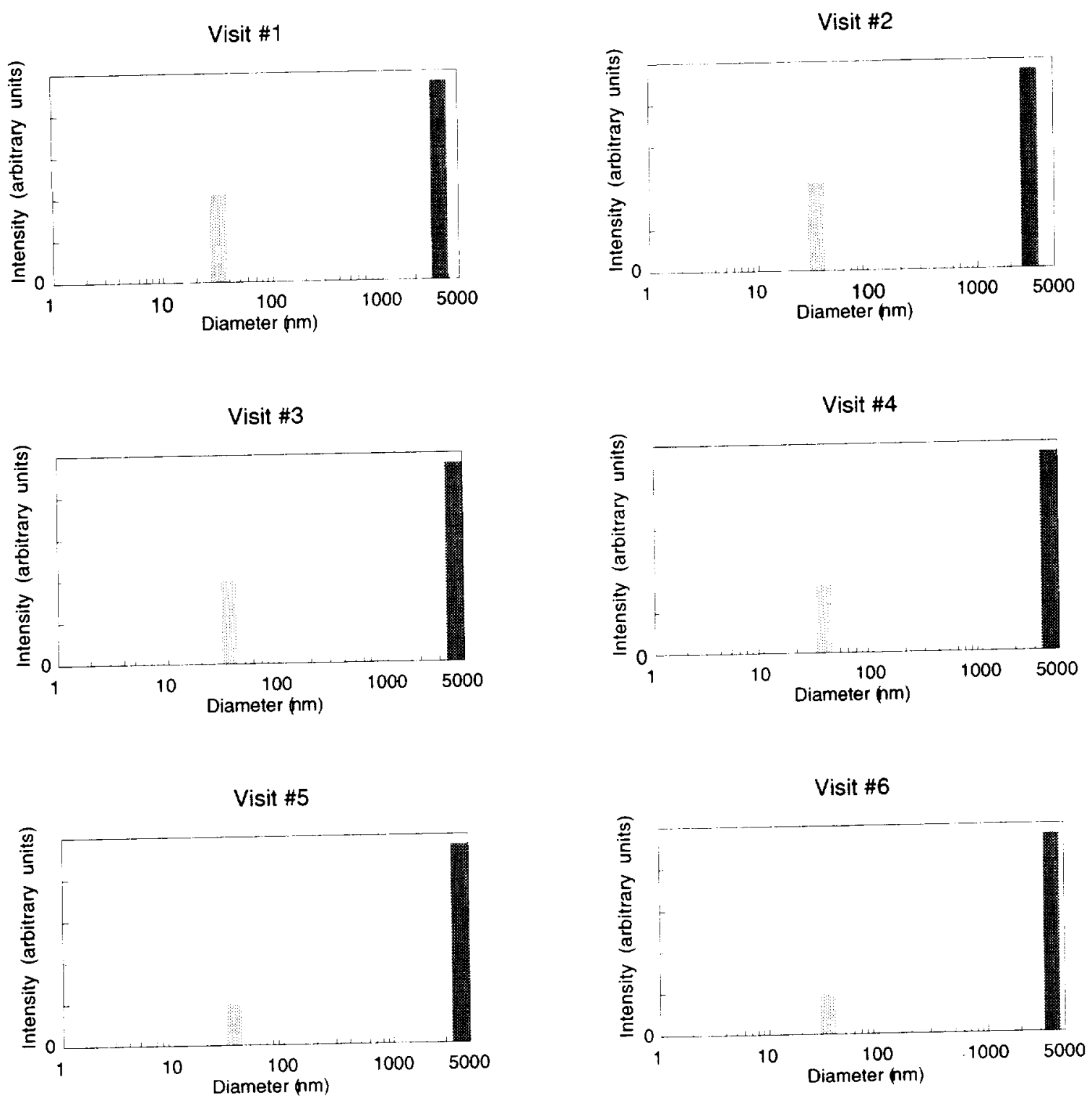

Visit \#7

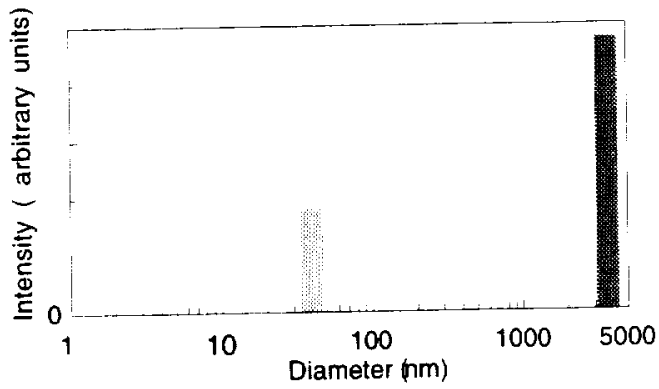

Figure 9. Size distribution using double exponential analysis of protein crystallines during patient's return visits.

-10w Component (Aggregates and higher molecular weight complexes) 


\section{DISCUSSION/MODEL}

At a first glance the uncertainties $7 \%$ (fast component) and $17 \%$ (slow component) in data reproducibility reported above appear large. This should be expected because the ocular tissue is far from an ideal solution as it contains three different types of proteins $(\alpha, \beta$, and $\gamma$ crystallins), their aggregates, and other higher molecular weight protein complexes. Furthermore, these moieties are not completely spherical. We carried out detailed investigation of the DLS data reproducibility under ideal conditions. We made DLS particle size measurements in separate aqueous solutions $(0.01 \% \mathrm{wt} / \mathrm{vol}$. concentration) and a mixture of $50 \pm 5 \mathrm{~nm}$ and $930 \pm 10 \mathrm{~nm}$ standard spherical particles of polystyrene. The binary mixture of these particles approximately mimics the polydispersity conditions encountered in the lens. The smaller and larger particles represent the fast $\alpha$-crystallines and the slower aggregates, complexes etc. respectively. In a well controlled DLS experiment of homogeneous solutions of small spherical particles of diameter $(50 \pm 5) \mathrm{nm}$, at a delay time of 10-2520 microseconds, we obtain an average value of $(42.49 \pm 1.45) \mathrm{nm}$. This value is reproducible within an uncertainty of $3.5 \%$. For a monodisperse system of $(930 \pm 93) \mathrm{nm}$ diameter particles, at a delay time of 100-23200 microseconds we recover size (diameter) value of $934.51 \pm 89.48 \mathrm{~nm}$ (within $10 \%$ ).
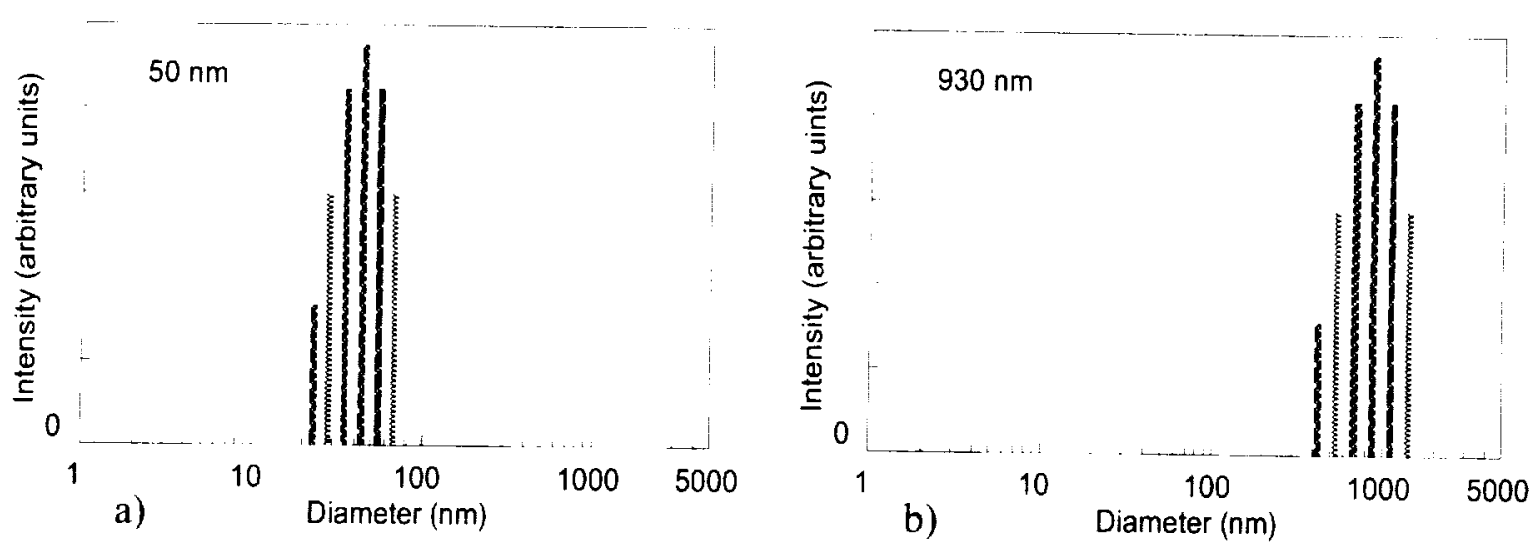

Size distribution of monodisperse particles using exponential sampling analysis.

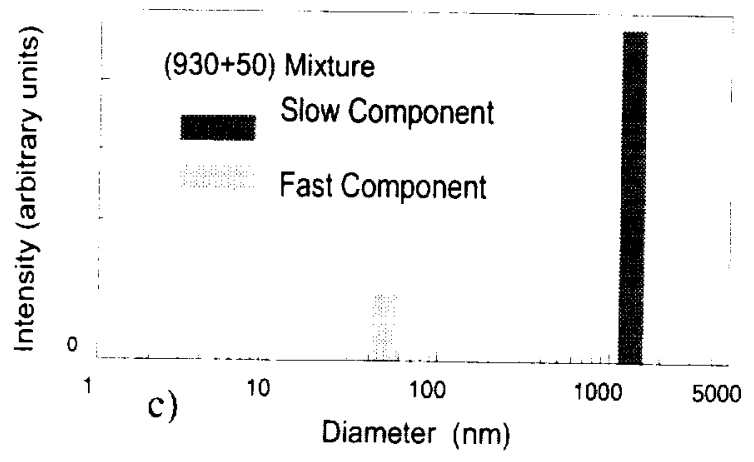

Figure 10. Size distribution of a binary mixture of particles using double exponential analysis of a cataract model. 
These measurements were made in 5 seconds duration time. A duration time of 30 seconds results in better statistics. But in the interest of patient safety we prefer 5 second runs. For the binary mixture the fast component is recovered as $50.90 \pm$ $3.60 \mathrm{~nm}$ (within $7 \%$ ) and the slow component as $1088 \pm 170 \mathrm{~nm}$ (within 15\%). The goodness of fit plots (figure 11 ) show that both the human lens data and the model experiment data is adequately described by the double exponential method. Furthermore data can be analyzed by both double exponential and multiple exponential schemes as show in figure 10 . This simple model suggests that the uncertainties in the reproducibility of DLS data from the ocular tissue is very reasonable considering the statistical nature of the scattered photons and a complex mix of different proteins and their aggregates or protein complexes.

a)

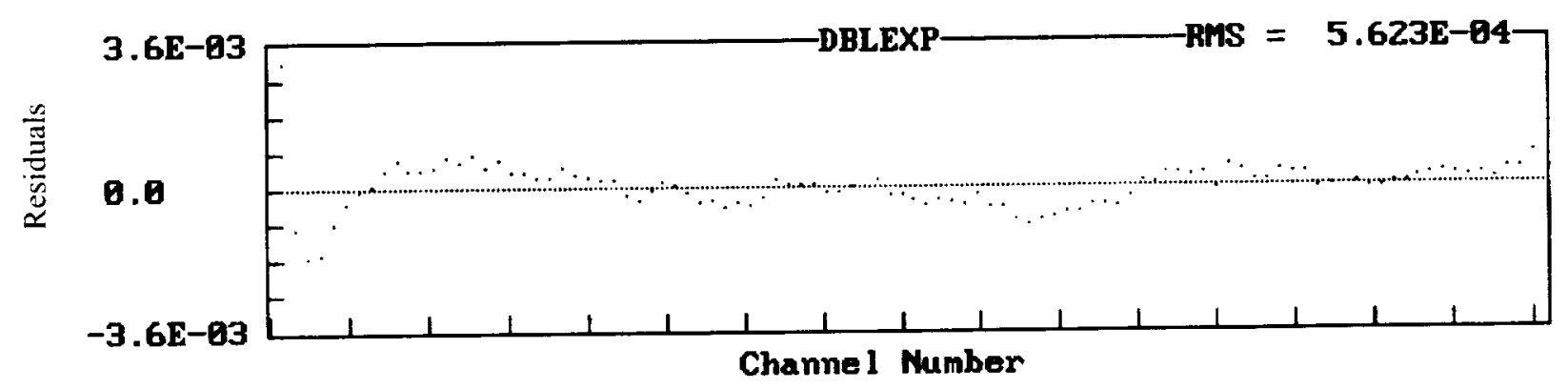

b)

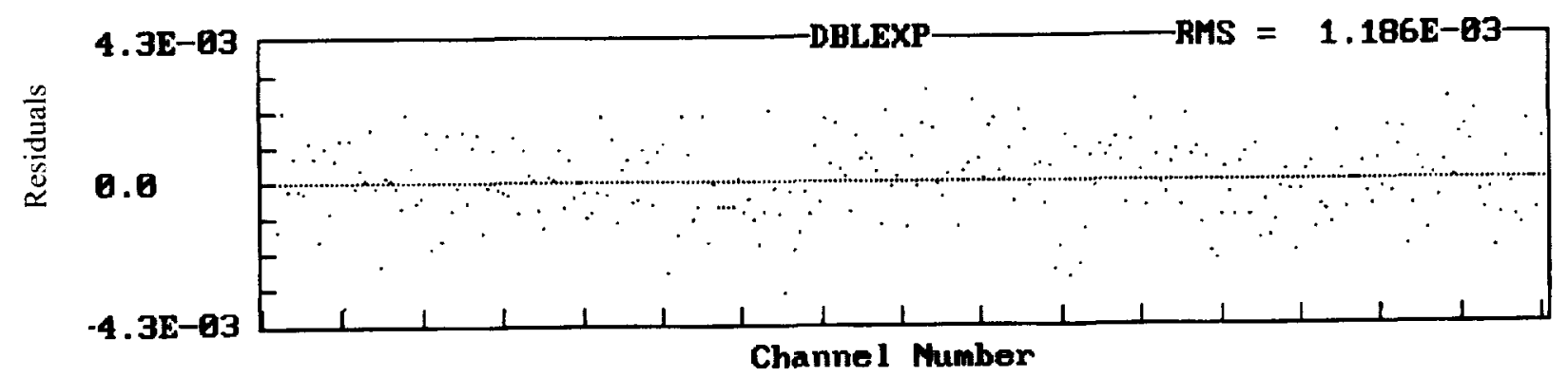

Figure 11. Residuals (Goodness of fit) plot.

a) Patient's typical eye data. b) Polydisperse model mixture. 


\section{CONCLUSIONS}

A new instrument combining DLS with corneal topography is found to be very sensitive and reliable in going back to the same location in the lens during patient's repeat visits. The unambiguous data obtained in this study is important in planning a longitudinal study of anti-cataract drug(s) screening soon to begin at NEl. A model based on observed polydispersity of the lens proteins describes the statistical uncertainties in the measurements. Finally, the patient did not report any discomfort during or after the DLS measurements.

\section{REFERENCES}

1. Foster, A., "Cataract-a global perspective: output, outcome and outlay," Eye 13, pp. 449-459, 1999.

2. Rubin, G.S., "What the aging are up against (visually)," $15^{\text {th }}$ Biennial Eye Research Seminar, Research to Prevent Blindness. 9-10, Sept. 26, 1995.

3. Owsley, C., "The Aging Driver," $15^{\text {th }}$ Biennial Eye Research Seminar, A compilation of papers presented at Research to Prevent Blindness Science Writers Seminar in Ophthalmology, 11,12, 1999.

4. Kupfer, C., Bowman Lecture, "The conquest of cataract: a global challenge," Trans. Ophthalmol. Soc. UK, 104, 1, 1984. 5. Chylack, L., L., M.E., Khu, P., Kashiwagi, T., Sperduto, R., "Lens opacities classification system II (LOCS II)," Clinical Ophthalmology, 107, pp. 991-997, 1989.

6. Benedek, G., Chylack, L., Libondi, T., Magnante, P., Pennet, M., "Quantitative detection of the molecular changes associated with early cataractogenesis in the living human lens using quasi-elastic light scattering," Curr. Eye Res., 1421. 1987.

7. Ansari, R.R., and Suh, Kwang I., DellaVecchia, M.A., and Dubin, S., "Ophthalmic Diagnostics Using a new Dynamic Light Scattering Fiber Optic Probe," Proc. Lasers in Ophthalmology III, SPIE vol. 2632, 1995.

8. Rovati, L., Frnakhauser II, F., Rick, J., "Design and performance of a new ophthalmic instrument for dynamic light scattering in the human eye," Rev. Sci. Instrum., 67, 2165, 1995.

9. Ansari, R.R., and Datiles, M.B., "Use of Dynamic Light Scattering and Scheimpflug Imaging for the Early Detection of Cataracts." J. Diabetes Technology \& Therapeutics, vol.1. Number 2, pp. 159-168, June 1999.

10. Ansari, R.R., Suh, K.I., Arabshahi, A., Wilson, W.W., Bray, T.L., and DeLucas, L.J., "A Fiber Optic Probe for Monitoring Protein Aggregation, Nucleation and Crystallization," J. Crystal Growth, 168, pp. 216-226, 1996.

11. Mattioli, B., and Tripoli, N.K., "Corneal Geometry Reconstruction with the Keratron Videographer," Optometry and Vision Science, vol. 74, no. 1, pp. 881-894, Nov., 1997.

12. Benedek et al., "Observation of Protein Diffusivity in Inact Human and Bovine Lenses with Application to Cataract." Investigative Ophthalmology, 14 (6), pp. 449-456, 1976.

13. Datiles, M., Magno, B., Friedlin, V., "Study of nuclear cataract progression using the National Eye Institute Scheimpflug System," Brit. J. Ophthalmol, 79, pp. 527-534, 1995. 
Public reporting burden for this collection of information is estimated to average 1 hour per response, including the time for reviewing instructions, searching existing data sources, gathering and maintaining the data needed, and completing and reviewing the collection of information. Send comments regarding this burden estimate or any other aspect of this

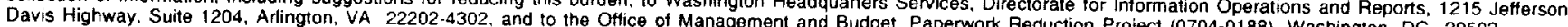

\begin{tabular}{|l|l|l}
\hline 1. AGENCY USE ONLY (Leave blank) & 2. AEPORT DATE & 3. REPORT TYPE AND DATES COVERED
\end{tabular}

\section{TITLE AND SUBTITLE}

September 2000

Final Contractor Report

A New Clinical Instrument for the Early Detection of Cataract Using Dynamic

Light Scattering and Corneal Topography

6. AUTHOR(S)

Rafat R. Ansari, Manuel B. Datiles, III, and James F. King

7. PERFORMING ORGANIZATION NAME(S) AND ADDRESS(ES)

National Center for Microgravity Research

21000 Brookpark Road

Cleveland, Ohio 44135

5. FUNDING NUMBERS

SPONSORINGMONITORING AGENCY NAME(S) AND ADDRESS(ES)

National Aeronautics and Space Administration

John H. Glenn Research Center at Lewis Field

Cleveland, Ohio 44135-3191

WU-101-51-00-00

NCC $3-544$

B. PERFORMING ORGANIZATION REPORT NUMBER

E-12161

10. SPONSORING/MONITORING AGENCY REPORT NUMBER

NASA CR-2000-209955

11. SUPPLEMENTARY NOTES

Rafat R. Ansari, National Center for Microgravity Research, 21000 Brookpark Road, Cleveland, Ohio 44135;

Manuel B. Datiles, III, National Eye Institute/NIH, Building 10, Room 10N226, Bethesda, Maryland 20892;

James F. King, Dynacs Engineering Company, Inc., 2001 Aerospace Parkway, Brook Park, Ohio 44142. Project Manager,

Bhim S. Singh, Microgravity Science Division, NASA Glenn Research Center, organization code 6712, (216) 433-5396.

12a. DISTRIBUTION/AVAILABILITY STATEMENT

Unclassified - Unlimited

Subject Categories: 12, 16, 43, 52 and 74

Distribution: Nonstandard

This publication is available from the NASA Center for AeroSpace Information. (301) 621-0390.

13. ABSTRACT (Maximum 200 words)

A growing cataract can be detected at the molecular level using the technique of dynamic light scattering (DLS). However, the success of this method in clinical use depends upon the precise control of the scattering volume inside a patient's eye and especially during patient's repeat visits. This is important because the scattering volume (cross-over region between the scattered light and incident light) inside the eye in a high-quality DLS set-up is very small (few microns in dimension). This precise control holds the key for success in the longitudinal studies of cataract and during anti-cataract drug screening. We have circumvented these problems by fabricating a new DLS fiber optic probe with a working distance of $40 \mathrm{~mm}$ and by mounting it inside a cone of a corneal analyzer. This analyzer is frequently used in mapping the corneal topography during PRK (photorefractive keratectomy) and LASIK (laser in situ keratomileusis) procedures in shaping of the cornea to correct myopia. This new instrument and some preliminary clinical tests on one of us (RRA) showing the data reproducibility are described.

14. SUBJECT TERMS

Cataract: Ophthalmology: Dynamic light scattering; Corneal topography; LASIK

\begin{tabular}{|c|c|c|}
\hline $\begin{array}{c}\text { 17. SECURITY CLASSIFICATION } \\
\text { OF REPORT } \\
\text { Unclassified }\end{array}$ & $\begin{array}{c}\text { 18. SECURITY CLASSIFICATION } \\
\text { OF THIS PAGE } \\
\text { Unclassified }\end{array}$ & $\begin{array}{c}\text { 19. SECURITY CLASSIFICATION } \\
\text { OF ABSTRACT } \\
\text { Unclassified }\end{array}$ \\
\hline
\end{tabular}

NSN 7540-01-280-5500 


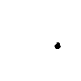


